\title{
Assessment of Knowledge, Attitude and Practice on Pulmonary Tuberculosis Among Heads of House Hold in Diga Town, Oromia, Ethiopia, 2019
}

\author{
Edosa Amente Gutema ${ }^{1}$, Zelalem Keba Babure ${ }^{2}$, Frezer Bekele Gudeta ${ }^{3}$ \\ ${ }^{1}$ Ethiopian Public Health Institute, Addis Ababa, Ethiopia \\ ${ }^{2}$ East Wollega Zone Health Office, Nekemte, Ethiopia \\ ${ }^{3}$ Ethiopian Public Health Institute, Addis Ababa, Ethiopia
}

Email address:

idoamante07@gmail.com (E.A. Gutema)

To cite this article:

Edosa Amente Gutema, Zelalem Keba Babure, Frezer Bekele Gudeta. Assessment of Knowledge, Attitude and Practice on Pulmonary Tuberculosis Among Heads of House Hold in Diga Town, Oromia, Ethiopia, 2019. Science Journal of Public Health.

Vol. 9, No. 5, 2021, pp. 142-148. doi: 10.11648/j.sjph.20210905.11

Received: August 12, 2021; Accepted: August 30, 2021; Published: September 6, 2021

\begin{abstract}
Ethiopia ranked $7^{\text {th }}$ among the 22 high burden globally. TB was the $3^{\text {rd }}$ leading cause of hospitalization and the $1^{\text {st }}$ leading cause of death in Ethiopia. Lack of appropriate knowledge and attitude of peoples about PTB affects the health seeking of patients and sustain the transmission of the disease. Objectives of this study was to assess knowledge, Attitude and practice of heads of households of the community towards PTB. A community based cross-sectional study was conducted in Diga town, East Wollega, Oromia, Ethiopia, 2019. A pre-tested structure questionnaire was prepared to obtain necessary information after getting both written and verbal consent from concerned bodies. The data was collected by using quantitative methods. The collected data was checked daily for the completeness and consistency calculation of proportion and other appropriate statistical tests was done and interpretation was reached accordingly. A total of 280 respondents with response rate of $97.6 \%$ and mean age of 32.9 years were included in the study. Cough more than two weeks $69.4 \%$ was the most common symptom known by the respondents. $88.2 \%$ were aware that TB could spread to others $69.8 \%$ air borne transmission. $45.4 \%$ of the participants aware that TB is caused by bacteria/germ. $53.2 \%$ of the respondents had good knowledge and $52.6 \%$ had poor attitude $57.5 \%$ respondents had good practice as well. The study participants had good knowledge of TB but there were several misconceptions regarding the cause of TB that need to be clarified. Community based programs on TB awareness need to be increased among young people and less educated groups. The study participants had good attitude and good practice.
\end{abstract}

Keywords: Knowledge, Attitude, Practice, Anti-TB, Treatment

\section{Introduction}

Tuberculosis is a chronic communicable disease caused by mycobacterium tuberculosis complex. It usually affects the lung but almost all organs can be affected, hence it is classified as pulmonary tuberculosis which account $80 \%$ and extra pulmonary tuberculosis (EPTB) in $20 \%$ of cases [1].

It is transmitted by inhalation of droplets, excreted via coughing, sneezing or speaking. Co infection with HIV/AIDS, malignancies, chronic disease, treatment with steroid and malnutrition are important risk factors for development of the disease. Productive cough of whitish or blood streaked sputum that persists more than 2 weeks, intermittent fever, night sweating, loss of appetite, and weight loss are the important clinical features. It is diagnosed by acid fast bacilli microscopy of the sputum like the other communicable disease it is preventable and treatable disease [1].

Good public awareness of PTB, its cause, sputum, mode of transmission, treatment and prevention is considered to be important for prompt health core seeking adherence to treatment and reduction of stigma associated with the disease. Nine million new cases of tuberculosis (TB) were diagnosed worldwide in 2017 and in the same year TB has killed 1.8 million people. In Sub Saharan Africa TB cases have markedly increased as a consequence of the HIV epidemic [3].

Almost three million deaths occur annually making TB the 
foremost cause of from a single pathogen in world. In Africa region the annual tool amount to 60,000 accounting over $20 \%$ of all death. About $15 \%$ of the three million deaths in world occur in children [2]. In East Asia 1.2 million deaths were due to TB case [3].

In Ethiopia TB is the first leading cause for Hospital death [4]. The highest, about (75\%) of morbidity and mortality rate are in age group 15-50 years [2], contributing significantly to decrees in production, Loss of spouse and thus to poor health and delayed development to surviving children [5]. In Ethiopia like other developing countries TB has long been known as major public health problem and still remains among the leading cause of morbidity and mortality [4]. Ethiopia attempts to control TB instituted by the ministry of health in the early 1960s with the establishment of TB center and sanitariums in urban in country, these had partially no impact in reducing the trend [6].

It is also argued that programmers with high defaulter rate might worsen the Epidemiological situation of TB, by contributing to the pool of chronic patients, than would be expected in situation without any attempt to treatment $[7,9]$.

Prompt diagnosis and early treatment of Tuberculosis (TB) cases is an important strategy in TB prevention and control. Thus, passive case finding of TB suspects, sputum examination for diagnosis, and prompt treatment using Directly Observed Treatment Short Course (DOTS) are key elements in the national guideline for TB control program [10].

Despite the introduction of directly observed treatment short course (DOTS) strategy, TB is still the leading cause of death in the developing world particularly is sub Saharan Africa [2]. According to the world health organization (WHO), worldwide 8.4 million people develop active TB every year and 2.3 million people die of its, of these mortalities due to TB in Africa is 10 times more in Europe [3].

Ethiopia ranked $7^{\text {th }}$ among the 22 high TB burden country globally. According to report from ministry of health TB was the $3^{\text {rd }}$ leading cause of hospitalization and the 7 th leading cause of hospital death [4]. WHO estimated the incidence of TB in Ethiopia were 365 people per 100,000 population per year and the incidence of smear positive pulmonary TB was 155 per 100,000 people per year [5].

Studies from the developing country have shown that delayed care is closely related with patients' demographic characteristics [8] knowledge of TB and traditional believes [9], the use of multiple alternative traditional care [10] and fear of stigmatization [11] lacking knowledge of TB among the general population could contribute to prolonged delayed in seeking TB care [12]. The ancient disease tuberculosis had been known as major cause of human suffering and death for centuries [15-17]. Although this was more so before the discovery of anti-tuberculosis drugs [16], tuberculosis (TB) still remains a major public health problem all over the world particularly in developing countries [18].

Effort to control TB globally particularly in developing countries have so far failed. The main reason for failure includes inadequate case management and over reliance on BCG [2]. In 1993, WHO has declared that TB is a global emergency and adopts a new strategy and framework for effective TB control directly observed treatment, short course [23].

The fact which was generated from this study will have great importance for various governmental and nongovernmental organizations and institutions, policy makers, stake holders, helping students and other researchers who are interested to another study.

\section{Objectives}

\subsection{General Objective}

To assess the knowledge attitude and practice of heads of house hold of the community towards PTB in Diga town in, West Ethiopia, 2019.

\subsection{Specific Objective}

a) To assess knowledge of heads of households towards PTB in Diga town, West Ethiopia, 2019.

b) To assess their attitude towards PTB and PTB patients in Diga town, West Ethiopia, 2019.

c) To assess practice on prevention and control of PTB patients in Diga town, West Ethiopia, 2019.

\section{Methodology}

\subsection{Study Area and Period}

The study was conducted in a specific area in, kebele 2 Diga town, East Wollega, Oromia, Ethiopia from May 1/2019 up to June 10/2019. Nekemte town "is a Zonal town of east Wollega in Oromia Region which is found $332 \mathrm{Km}$ west of Addis Ababa and Diga town is about $12 \mathrm{~km}$ from Nekemte town. It has an elevation of $2088 \mathrm{M}$ above sea level. Currently the town has 1 health center, 5 private clinic, 6 drug dispensary.

The total population of the Diga town according to 2007 census male 39,167 and female 37,650 Total 76,817. Currently the total population of Diga town is 140,000 . The town has 2 kebeles.

\subsection{Study Design}

Quantitative, descriptive community based cross sectional study was under taken.

\subsection{Population}

\subsubsection{Source Population}

Source population was 463 houses hold in " 02 kebele of Diga town.

\subsubsection{Study Population}

Heads of house hold ( 18 years and above) of " 02 kebele of Diga town.

\subsection{Inclusion and Exclusion Criteria}

\subsubsection{Inclusion Criteria}

Permanent members of the kebele who are heads of households (Who live for $>=6$ month). 


\subsubsection{Exclusion Criteria}

Those children $(<18$ years old $)$, unconscious, unable to hear or speak, those with psychiatric disorder.

\subsection{Sample Size Determination}

The sample size was determined by using a standard formula for single population proportion. A non-respondent rate of $5 \%$ was calculated and this number was included in the study by using $95 \%$ confidence on interval and 5\% margin of error the sample size was determined as follows.

$$
\begin{gathered}
\mathrm{n}=\frac{(\mathrm{z} \propto / 2) 2 \mathrm{p}(1-\mathrm{p})}{\mathrm{d} 2} \\
\mathrm{n}=\frac{(1.96) 2(0.21)(0.79)}{(0.05) 2}=255
\end{gathered}
$$

Therefore $\mathrm{No}=(1.96)^{2}(0.21)(0.79) /(0.05)^{2}=255$

Non-respondent rate $=255 \times 10 \%=25$

Finally, $\mathrm{No}=255+25=280$

Hence a total of 280 sampling was taken

\subsection{Sampling Procedure}

The town has 2 kebeles, and kebele 2 was selected by lottery method and then the households were selected by systemic random sampling technique.

The sampling interval was determined by dividing $\mathrm{N} / \mathrm{n}$ $(463 / 280=1.66=2)$.

From number 01 and 02 the one was selected randomly, then it was the $1^{\text {st }}$ how hold to be included and every 02 interval was interviewed heads of households in the selected house were identified for the interview.

\subsection{Data Collection}

Quantitative data was obtained using pretested, structure questionnaire prepared in English and then translated to Afan Oromo and Amharic. This interview was administered for heads of house hold, if there are no heads of house hold during the visit, the next most senior person for the house hold whose age is above 15 years were interviewed. If no one is present in the selected house hold a $2^{\text {nd }}$ and of net yet found, another visit was made. After $3^{\text {rd }}$ visit recorded as non-response.

Respondents' socio demographic characteristics (age, gender, educational, marital status, family size and income), PTB knowledge (it causes, transmission, sign and symptom, prevention and treatment) was collected through interviews.

\subsection{Study Variables}

a) Dependent variables

1) Knowledge about PTB

2) Attitude towards PTB and PTB patients

3) Practice towards PTB control and prevention

b) Independent variable

Socio demographic variables: age, sex, marital status, family income, educational status, family size.

\subsection{Data Quality Assurance}

The questionnaire which is first prepared in English translated to Afan Oromo by language experts. The translated questionnaire was retranslated to English. The structured questionnaire was pretested on the heads of house hold using $5 \%$ of sample size which is not included in the sample for classify and cultural acceptability. The principal investigator conducts this and the necessary modifications were done when appropriate, each day the data was checked for completeness and consistency refilled if any.

Data processing and analysis

The data was entered into Epi info 3.1 and analyzed using SPSS version 20. Descriptive statistics was carried out to describe major variable of the study. The results presented by tables, figure and charts.

\subsection{Ethical Consideration}

Individual consent was obtained from each respondent and then, also told have the right to give up the interview at any time when he /she wish.

\section{Results}

\subsection{Socio-demographic Characteristics of the Study Participants}

A total of 280 study participants (39.3\% males and $60.7 \%$ females; age started from 18 and above were interviewed and the response rate was $97.6 \%$. The mean age of the study participants was 32.9 years $(\mathrm{SD}=10.3)$. About $(75.8 \%)$ were educated. With regard to ethnic composition, $280(100 \%)$ were Oromo. Majority of the study participants $(66.8 \%)$ were followers of Orthodox religion, $10.7 \%$ were Muslims and $22.5 \%$ were protestants. $75.4 \%$ of respondents were married. About $15.4 \%$ of respondents were single. $30.8 \%$ had received primary education. $40.3 \%$ had between 500-1000 ETB monthly incomes.

\subsection{Knowledge of Community Towards Tuberculosis}

Knowledge status of community was assessed by general knowledge by asking question to identify it. These questions were about as bacteria is a cause of $\mathrm{TB}$, responding the sign and symptom of Tuberculosis are cough, weight loss of appetite cough for two or more weeks, weight loss, loss of appetite \& febrile Ness, knowledge as TB is communicable disease or not and (mode of transmission, air borne, living together with TB affected patients, hereditary, sexual intercourse, using common utensils). (4) Knowing that TB is preventable or not and ways of correct prevention (covering mouth and nose when coughing or sneezing, house ventilation, immunization of children, avoid sharing cups, using separate rooms, opening window of public transport service and avoiding hand shaking). (5) Able to mention the correct risk factors (overcrowding, malnutrition, immune suppression) (6) ways of treatment (traditional method, modern therapy, pray and others). All questions were rated 
and that given to correct answers and zero was given for those whose responses was wrong.

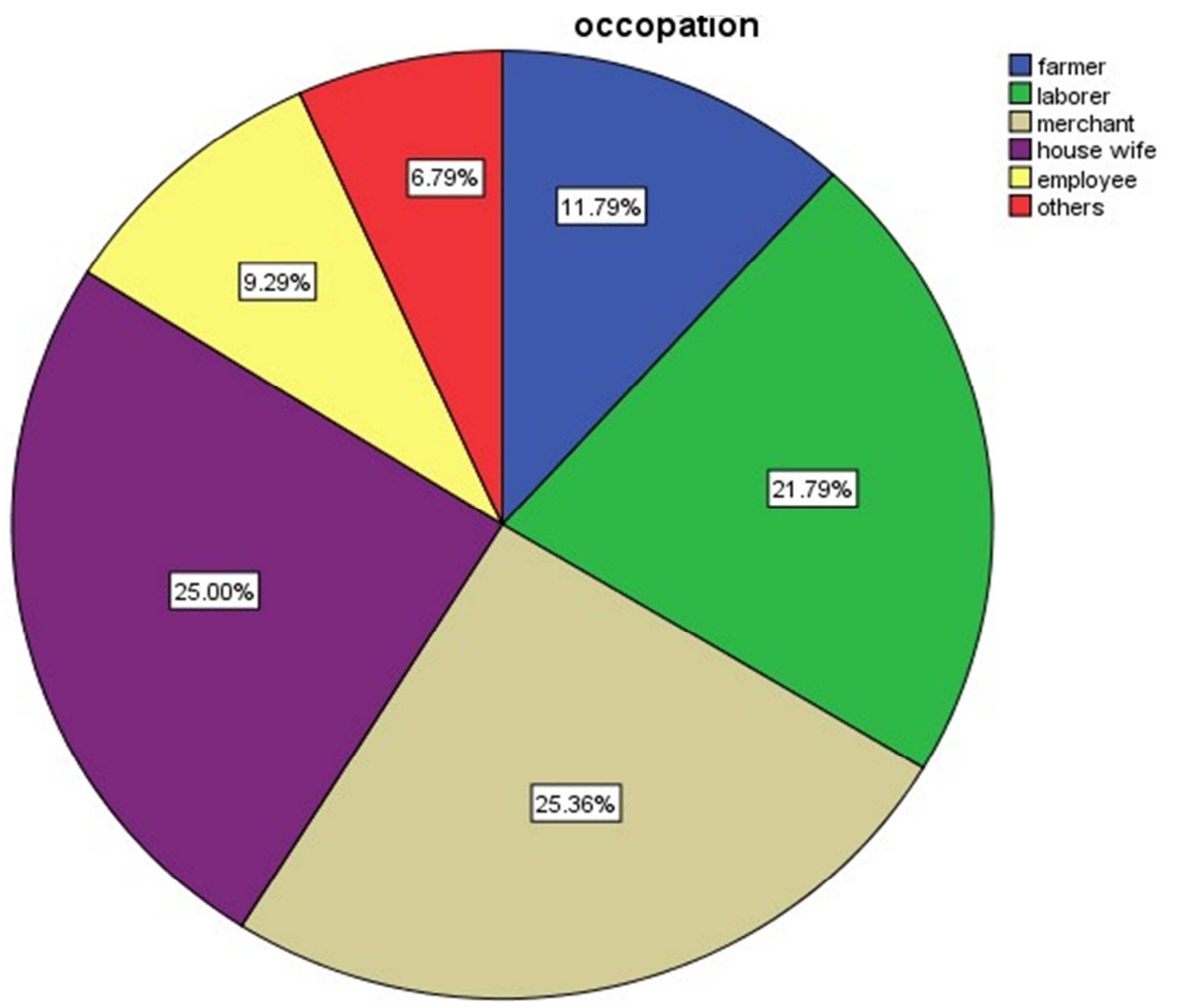

Figure 2. Distribution of occupations of the community in Diga town 2019.

educational status

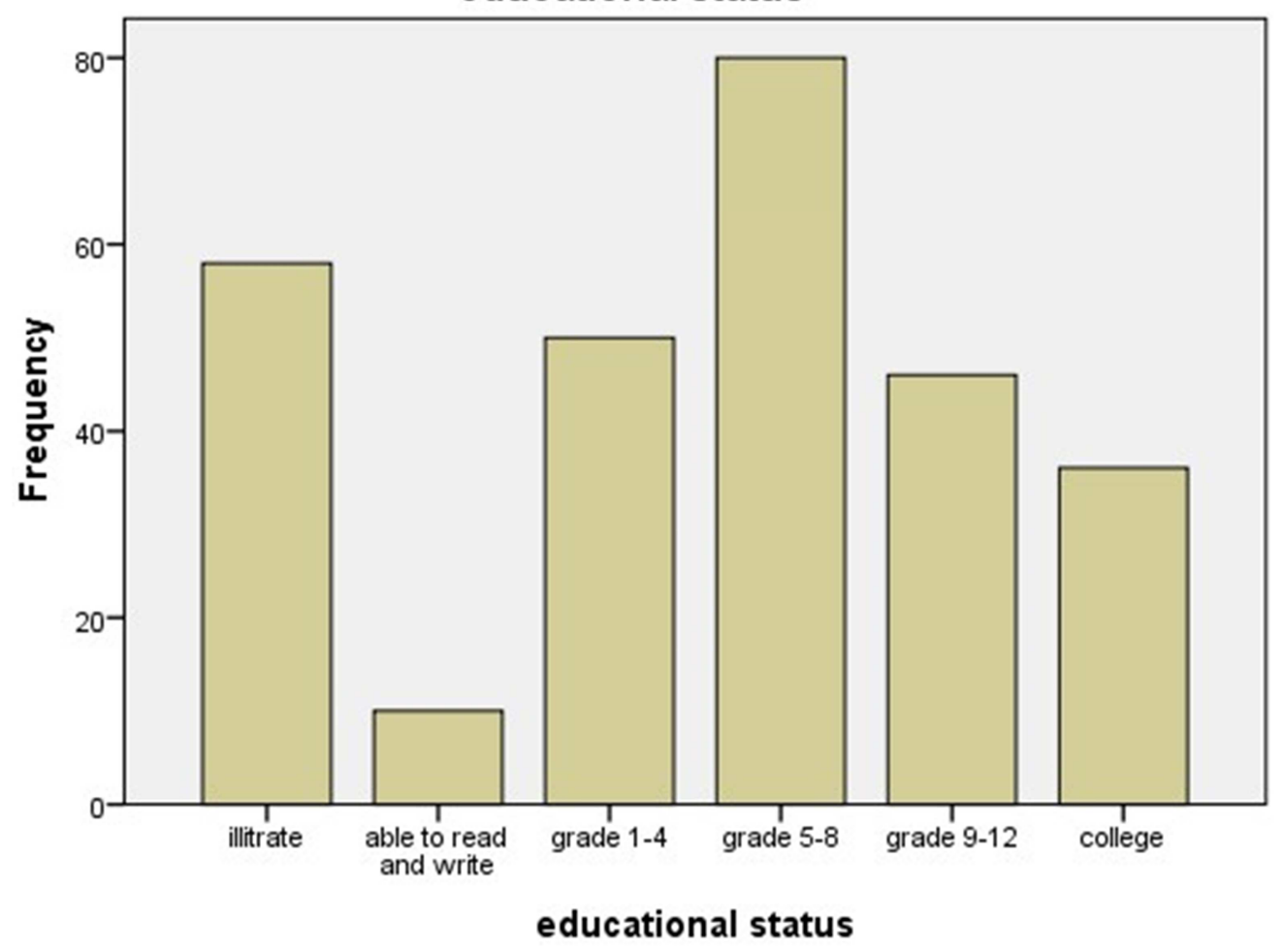

Figure 3. Educational status of the community in Diga town 2019. 


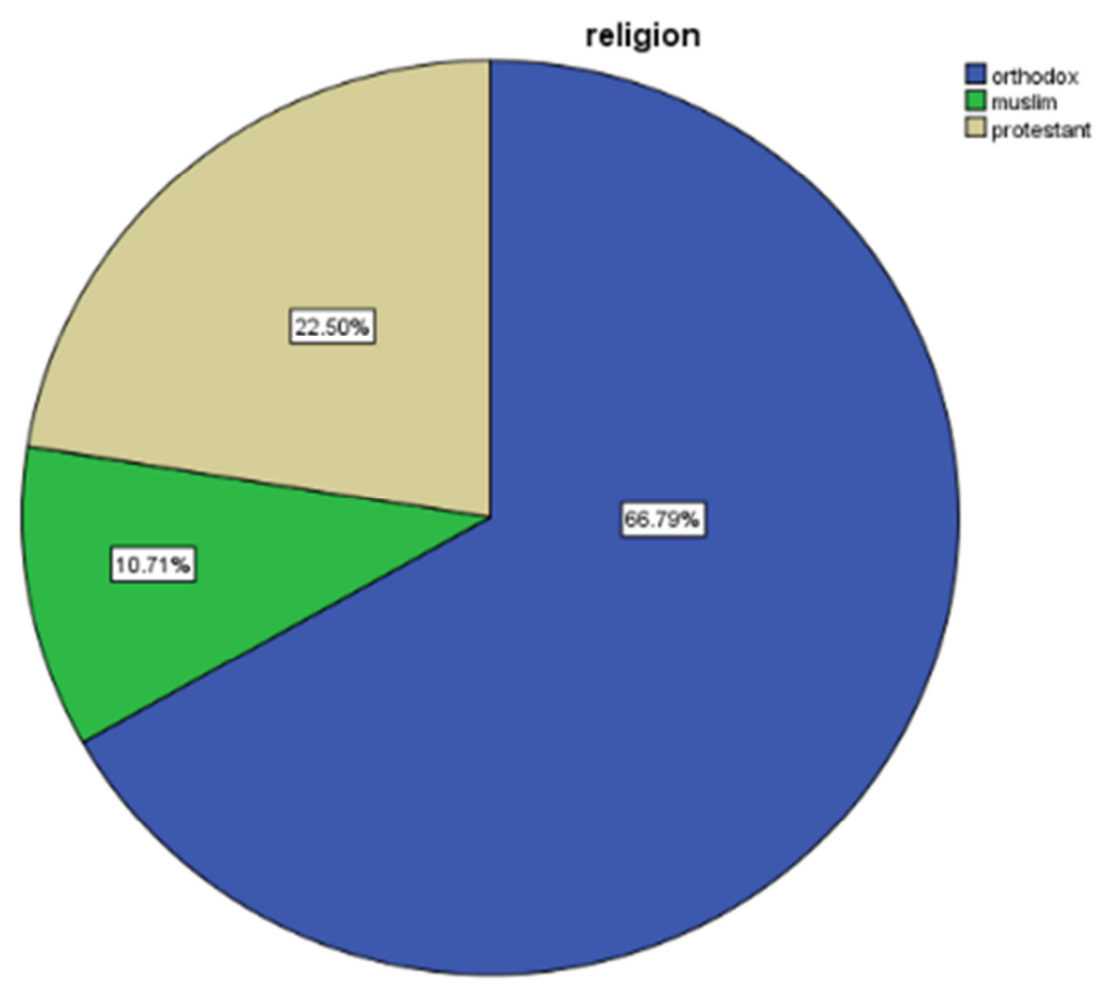

Figure 4. Religion of the respondents in Diga town, 2019.

Also, their answers were used to generate scoring range from 0 to 25 . At the end, the composite result was dichotomous by mean of 13.6 as cutting point value and the score more than mean value was coded as 2 by showing high knowledge of Tuberculosis and score less than mean value was coded as 1 showing low knowledge of TB in that community. The mean knowledge of TB was 13.6. About 131 (46.8\%) participants had poor on knowledge about TB and $149(53.2 \%)$ have good general knowledge on TB.

\subsection{Knowledge About the Source of Information, Cause, Symptoms, Mode of Transmission, Prevention and Treatment of $T B$}

All $(100 \%)$ of study participants had information regardless of their sex. Mass media was the main source of information, which is $179(70.3 \%)$ and $75(26.7 \%)$ from health institution and $8(2.9 \%)$ from others. From all respondents, $112(40 \%)$ answered "M. tuberculosis" or "bacteria/germs" on what they asked as "what is the cause of TB?" An answer of others were $127(45.4 \%)$, shortage of food $15(5.4 \%)$, punishment of god $13(4.6 \%)$ and don't know 13 (4.6\%).

Cough persisted for more than 2 weeks 111 (39.6\%) was the commonest symptom of TB reported. Respondents' responses regarding mode of transmission and preventive methods of TB are about $88.2 \%$ of our respondents known that $\mathrm{TB}$ is a communicable disease. About (69.8\%) of the study participants said that $\mathrm{TB}$ is transmitted through airborne. Covering mouth while coughing was mentioned as a means of preventing TB transmission by $52.0 \%$ of the study participants. $4.1 \%$ of the study participants mentioned "vaccinations of children "as the most important method of preventing and control of TB. About $58.8 \%$ of the study participants said that TB is treatable with modern drugs. From 280 study participants only $69(24.6 \%)$ have television and $106(37.9 \%)$ have radio.

The attitude of the community towards PTB

The overall attitude of the study participants was assessed by 6 questions (1) afraid of PTB patient s, (2) continue friendship or not with PTB patients, (3) provide care or not for PTB patients, (4) tell to others or not when develop PTB, (5) why don't tell (sham full, fear of stigmatization) and (6) allow or not daughters or sons to marry cured PTB patients.

From 280 study participants $124(44.3 \%)$ said yes and 102 (37.1\%) said no and 51 (18.3\%) said I don't know for the question "do you think all PTB patients have HIV/AIDS? "And for the question do you PTB patients 130 (46.4\%) said yes and $150(53.6 \%)$ said no. $83.9 \%$ of the study participants said we would continue friendship with PTB patients.

For the question would you provide care PTB patients 160 (57.1\%) said yes and 120 (42.9\%) said no and for the question would tell to others if you develop PTB $70 \%$ of the respondents said yes and $30 \%$ of the respondents said no. Also, their response to questions were added to generalize their attitude scoring from 0 to 17 . Finally, the dichotomous mean score was 7.5 as cut off point the score more than mean value was coded as 2 by showing high attitude on Tuberculosis and score less than mean value was coded as 1 showing low attitude of TB in that community. The mean attitude of TB was 7.5. About 147 (52.6\%) participants had poor attitude about TB and 133 (47.4\%) have good general attitude on TB.

Practice of the community was measured by the two 
question (1) what do you do if you get PTB patients and what do you do if you develop PTB. From 280 study participants $184(65.7 \%)$ said help him/her to get clinic or hospital, 34 (12.1\%) said help him/her to get Holley water, 36 (12.9\%) advise him/her to take home medications and $23(3.2 \%)$ do nothing. For the question what do you do if you develop PTB $154(55 \%)$ go to hospital, $52(18.6 \%)$ go to traditional healer, $46(16.4 \%)$ go to Holley water and 28 (10\%) keep secret. The response of the participants was scored zero to eight. The mean practice of the respondents was 4.6 . $57.5 \%$ of the respondents had good overall practice and $42.5 \%$ of the respondents had poor practice.

\section{Discussion}

All $(100 \%)$ of the current respondents have heard about TB. Similar findings were -reported from study that was conducted in Libya. [22]

In the current study, the main sources of information mentioned by the study participants were Mass media which is consistent with participants' mentioned mass media as major source of information about TB in study conducted in Shinnie town Somali Regional state. The similarity might be due to presence of mass media in the present study area as compared to the shinile town Somali regional study area [21, 22].

Higher proportion of the study participants have heard about $\mathrm{TB}, 40 \%$ of the participants correctly answered the cause of $\mathrm{TB}$ as being bacteria which is inconsistent with the findings of study conducted in Philippines which is only $4.4 \%$ [24].

Cold air $45.4 \%$ and shortage of food $5.4 \%$ were frequently mentioned as the cause of TB by the present study participants which is similar to the findings of studies conducted in South East Nigeria [13]. In the present study cough of 2 weeks and above was mentioned as symptoms of TB by most of study participants which is almost similar to study conducted in Libya and shinile town Somali region [21, 22].

In the present study most of respondents mentioned that covering mouth and nose during coughing and sneezing were mentioned as the most preventive measures of TB transmission which was coherent with study conducted in southeast Nigeria; this may be due to further expansion of mass media and HID given by University students and HEW $[21,25]$.

In this study, the study participants reported that $\mathrm{TB}$ is treatable and curable with modern drug: These findings are in line with other studies conducted in Itang special district, Gambella Region, South West Ethiopia [24]. In our study some respondents were of the opinion that TB can be caused by cold air.

\section{Strength and Limitations of the Study}

\subsection{Strength}

In this study, efforts were made to assess the knowledge of community towards TB which could support the TB control programs in Diga town.

\subsection{Limitation}

The study has potential limitations including lack of budget to include total woreda.

\section{Conclusion}

This study reveals that in general, knowledge about TB of the population was adequate. It also identified number of a few gaps in the area of cause, transmission, Prevention and treatment of tuberculosis.

Our study respondents indicated mass media as most frequent source of information. Hence, all measures should be taken to encourage HEW to do more on cause, preventive measure and to promote community knowledge. $53.2 \%$ of the study participants had good knowledge and $52.6 \%$ of the respondents had poor attitude. Among the study participants $57.5 \%$ had good overall practice.

\section{Recommendation}

a) Therefore Government, Nongovernmental Organization should promote specialized educations programs for community members to reduce knowledge gap, attitude and practice.

b) Training given to participants or community should focus to change knowledge, attitude and practice and set measures.

c) Health facilities should make available within their system TB infection control guidelines and standardized written policies so that the community will know how to prevent and control the disease.

\section{Acronyms}

AIDS=Acquired Immune Deficiency Syndrome, $\mathrm{CBE}=$ Community Based Education

DOTS=Direct Observed Treatment short course EPTB $=$ Extra Pulmonary Tuberculosis

$\mathrm{HIV}=$ Human Immunodeficiency Virus, KAP=Knowledge, Attitude, Practice KM=Killo Meter

$\mathrm{PI}=$ Principal Investigator, $\mathrm{PTB}=$ Pulmonary Tuberculosis, SNNPR=Southern Nation National People Regional

$\mathrm{TB}=$ Tuberculosis, $\mathrm{WHO}=$ World Health Origination

\section{Declarations}

\section{Ethics Approval and Consent to Participate}

An individual participants' written consent for participation was obtained.

\section{Consent to Publish}

Not Applicable. 


\section{Competing Interests}

The authors declare that they have no competing interests.

\section{Funding}

The financial support for this research project was by PI and no any other financial support from anyone.

\section{Authors' Contributions}

EA conceived the study and wrote the proposal, facilitated data collection, analysis, data interpretation, drafted the final report write up and prepared manuscript. ZK and FB participated in developing the tools and data collection process and involved in report write up. All authors read and approved the final manuscript.

\section{Acknowledgements}

Heartfelt gratitude to supervisors, data collectors and study participants for their cooperation and support during the study period.

\section{References}

[1] Tizazu G: Anteneh T, internal medicine for health officer student, lecture note, Jimma University, 2014.

[2] WHO: Global TB control, surveillance, planning, financing, Geneva 2013.

[3] WHO: Global TB control, Geneva 2018.

[4] Federal Ministry of health and health related indicator planning and programming department, 2013.

[5] WHO, global TB control, Surveillance, planning and financing global TB report 2016.

[6] Lawn SD, Afful B, Acheampong JW, pulmonary TB; diagnostic delay in G. lnion adults, Int J Tuber lung dis 2018; 4 (12); 1190-1.

[7] Colebunders R, lambert Lm. Management of Co- infection with HIV and TB- Br med J, 2012; 324: 802-3.

[8] Hoa NP, Therson AE, long NH, Diwen VK, Knowledge of tuberculosis and associated health seeking behavior among rural Vietnamese adults with a cough for at least three weeks. Scand J public Health 2013 supply (62); 59-65.

[9] Shefty N Shamco. M Abbas, knowledge Attitude and practis regarding Tuberculosis among immigrants of Somalion Ethnic origin in London: A cross sectional study, commundis, and public health 2014: $6-7$ (1): 77-82.

[10] Wyss K. Kilima 9-lorenz N, costs of Tuberculosis for households and health care providers, in Dereselem: Tanzania trope med in health, 2011/6;60-8.
[11] Getahun $\mathrm{H}$ medical and social consequence of tuberculosis control in rural district in Ethiopia, Ethiopia medical Jornal 20094 (2): 174-178.

[12] Davery J, report on knowledge, attitude \& practice survey regarding TB, in Northern bihar India 13 April 2011.

[13] Jacob P. KAP of TB among maajai in Sinanjiro district, Tanzania 2010.

[14] CDC; morbidity and mortality report increase in African immigrants with TB voles, 51/No 39 Oct, 04, 2013.

[15] Muktar E, Assessment of KAP on pulmonary TB among adults in Arbaminch Town and Arbaminch Zuria Woreda, Gamo Gofa zone $<$ SNNPR, Ethiopia, extract No of Addis Abeba, August 2010. 74-89.

[16] Legese M. knowledge and perception of PTB in pastoral communities in the Middle and lower Awash valley of Afar region, Ethiopia public health 2010.

[17] EPHA: assessment of knowledge and perception of the community in Gilgel Gibe, extract No 6, Addis Abeba, Ethiopia, 2010.

[18] Mesfin M. community KAP on PTB and their choice of treatment supervisor in Tigray, Northern Ethiopia, health Dev. 2015, 19 (Special issue).

[19] Yigzaw T. introduction to research methodology, lecture note for health science students JImma University, 2014.

[20] A review of compliance to anti tuberculosis treatment and risk factors for defaulting treatment in Sub Saharan Africa. B Castelnuovo. Afr Health Sci. 2012 Dec; 10 (4).

[21] WHO (2012), Treatment of TB, Guide line for national program (2014) second edition pp 13-50.

[22] World Health organization (2014) manual for national TB and Leprosy control program in Ethiopia, 2014.

[23] Ministry of Health (2015) manual for national TB and leprosy control program in Ethiopia, 2015 pp 1-26.

[24] H. Kloos and Ahmed Zein (2012), Tuberculosis, In the ecology of Health and disease in Ethiopia.

[25] Dye C, Bassili A, Bierrenbach AL, Broekmans JF, Chadha VK, Glaziou $P$, et al., et al. Measuring tuberculosis burden, trends, and the impact of control program. Lancet Infect Dis 2013; 8: 233-43 http://dx.doi.org/10.1016/S14733099(07)70291-8 pmid: 18201929.

[26] Dyec, Garnelt GP: et al (2013) Dots a breakthrough in TB control world health, 2013; pp 14-15.

[27] Christopher Dye, Catherin J, watt and denial Bleed (2014), Low access to a highly effective therapy challenge for international TB control, Bulletin of WHO 2014, Vol 80 (6) pp 437-44.

[28] Demissie M, Kebede D. 2012, Defaulting from tuberculosis treatment Addis Ababa TB center and factors associated with it, Eth. Journal 2012, Co 132 pp 91-97. 